\title{
A Risk Prediction Model of Readmission for Chinese Patients after Coronary Artery Bypass Grafting
}

\author{
Guozhen Liu, Undergraduate, ${ }^{1}$ Yinghong Zhang, MD, PhD, ${ }^{1}$ Wen Zhang, $M D,{ }^{2}$ Yanhong Hu, Graduate, ${ }^{1}$ \\ Tiao Lv, Undergraduate, ${ }^{1}$ Hong Cheng, $\mathrm{MD}$, PhD, ${ }^{1}$ Jing Huang, $\mathrm{MD}^{3}$ \\ ${ }^{1}$ School of Medicine, Wuhan University of Science and Technology, Wuhan, Hubei, China; \\ ${ }^{2}$ Department of Cardiology, Wuhan Asian Heart Hospital, Wuhan, China; ${ }^{3}$ Wuhan Puren Hospital, Wuhan, Hubei, China
}

\section{ABSTRACT}

Background: Predictive models can be used to assess the risk of readmission for patients after coronary artery bypass grafting (CABG). However, the majority of the existing prediction models have been developed based on data of western population. Our objective was to develop and validate a risk prediction model for Chinese patients after CABG.

Methods: This study was conducted among 1983 patients who underwent CABG in Wuhan Asian Heart Hospital from January 2017 to October 2019. Pearson's chi-squared and multivariate logistic regression were performed to investigate the risk factors of readmission after CABG. The area under the ROC curve and Hosmer-Lemeshow test were used to validate the discrimination and calibration of the model, respectively.

Results: Six risk factors were predictive of readmission: age $\geq 65$ years (odds ratio $[\mathrm{OR}]=2.19 ; 95 \%$ confidence interval $[\mathrm{CI}]: 1.11-4.34 ; P=0.024)$, female $(\mathrm{OR}=2.46 ; 95 \% \mathrm{CI}$ : $1.26-4.80 ; P=0.008)$, private insurance $(\mathrm{OR}=4.23 ; 95 \% \mathrm{CI}$ : $1.11-16.11 ; P=0.034)$, diabetes $(\mathrm{OR}=2.351 ; 95 \%$ CI: 1.20 $4.59 ; P=0.012)$, hypertension $(\mathrm{OR}=2.33 ; 95 \% \mathrm{CI}: 1.16-4.66$; $P=0.017)$, and congenital heart disease $(\mathrm{OR}=6.93 ; 95 \% \mathrm{CI}$ : 2.04-23.52; $P=0.002)$. The area under the curve c-statistic was 0.876 in the derivation sample and 0.865 in the validation sample. Hosmer-Lemeshow test: $P=0.561$.

Conclusion: The risk prediction model in our study can be used to predict the risk of readmission in Chinese patients after CABG.

\section{INTRODUCTION}

Coronary heart disease is one of the most common heart diseases to harm people's health. Coronary artery bypass grafting (CABG) rapidly has improved in recent years and has become the effective treatment for coronary heart disease.

Received March 6, 2021; accepted April 5, 2021.

Correspondence: Wen Zhang, MD, Department of Cardiology, Wuban Asian Heart Hopsital, Wuhan, China, 430022 (email: zw21320431@sina.com).
However, adverse cardiovascular and restenosis after surgery force patients to be readmitted to the hospital [Patchouli 2019; Alejandra 2019]. Therefore, a risk prediction model of readmission may be useful for physicians and hospitals to take comprehensive interventions in the early stages [Kong 2012].

Previous studies mainly have focused on the investigation of risk factors related to CABG readmission [Shah 2019; Deng 2018; Price 2013; Han 2011], but few have established the models to predict the quantitative risk of CABG readmission [Woozy 2018; Bertillon 2018; Tam 2018]. Although predictive models have been developed in western countries like the United States and Canada, due to different epidemiological characteristics such as ethnicity, genetics and medical security system, these models cannot be well applied to Chinese or other Asian populations [Amati 2015; Yuan 2012].

In this study, we aimed to identify the risk factors associated with readmission after CABG in Chinese patients, then build the risk prediction model for readmission and validate it.

\section{MATERIALS AND METHODS}

Our study was approved by Wuhan University of Science and Technology (202042). All records of patients, who underwent CABG from January 2017 to October 2019 in Wuhan Asian Heart Hospital, were collected. Inclusion criteria: age $\geq 18$ years; patients underwent CABG. Exclusion criteria: patients with mental disorders; incomplete data. Among 1983 patients, 825 cases from January 2017 to December 2017 that underwent CABG were used as the derivation cohort, while 1158 cases from January 2018 to October 2019 were used as the validation cohort.

Among all variables, there were 13 preoperative variables and eight postoperative variables. Preoperative variables included age, gender, insurance type, diabetes, hypertension, hyperglycemia, congenital heart disease (atrioventricular septal defect, congenital coronary artery fistula, congenital cardiac diverticulum), history of angina, heart failure classification, coronary artery muscle bridge, valvular heart disease, previous cardial infarction, and obsolete brain infarction. Postoperative variables included abnormal liver function, abnormal renal function, neural effusion, ascending aorta dilation, cerebral artery stenosis, renal artery stenosis, vertebral artery stenosis, and arrhythmia. Readmission was defined as the patient's admission within 30 days after CABG. 
Data were analyzed using SPSS 27.0 software (IBM, Armonk, NY, USA). If data did not conform to normal distribution after inspected, then Mann-Whitney $U$ test and Pearson chi-square test were used. Discrimination and calibration were performed using the area under the ROC curve (AUC) and the Hosmer-Lemeshow test, respectively. Calibration degree is one of the best characteristics reflecting the prediction efficiency of the prediction model. Hosmer-Lemeshow goodness of fit test is generally used, and $P>0.5$ indicates good calibration degree [Jia 2019].

\section{RESULTS}

Among 1983 patients who underwent CABG, 1339 $(67.5 \%)$ were males and $644(32.5 \%)$ were females. The average age was 61.7 years (range, 24-95y). A total of 96 (4.8\%) patients were readmitted after surgery. Of these patients, five patients were readmitted more than one time, and five patients were readmitted within 72 hours after surgery. The common reasons for readmission were heart failure (22.9\%), arrhythmia (14.6\%), neural effusion (12.5\%), lung infection $(11.5 \%)$, surgical incision infection $(11.5 \%)$, cerebral infarction $(7.3 \%)$, acute coronary syndrome $(3.1 \%)$, and non-classifiable cause $(16.7 \%)$.

There were significant differences in eight variables between readmitted and non-readmitted patients, including age, gender, insurance type, diabetes, hypertension, congenital heart disease, history of angina, and abnormal renal function $(P<0.1)$. (Table 1$)$ Significant predictors of 30 -day readmission in the multivariate analysis included age $\geq 65$, female gender, private insurance, diabetes, hypertension levels 2 and 3 , and congenital heart disease. (Table 2)

The formula for calculating the risk of readmission was

Table 1. Baseline characteristics in derivation cohort

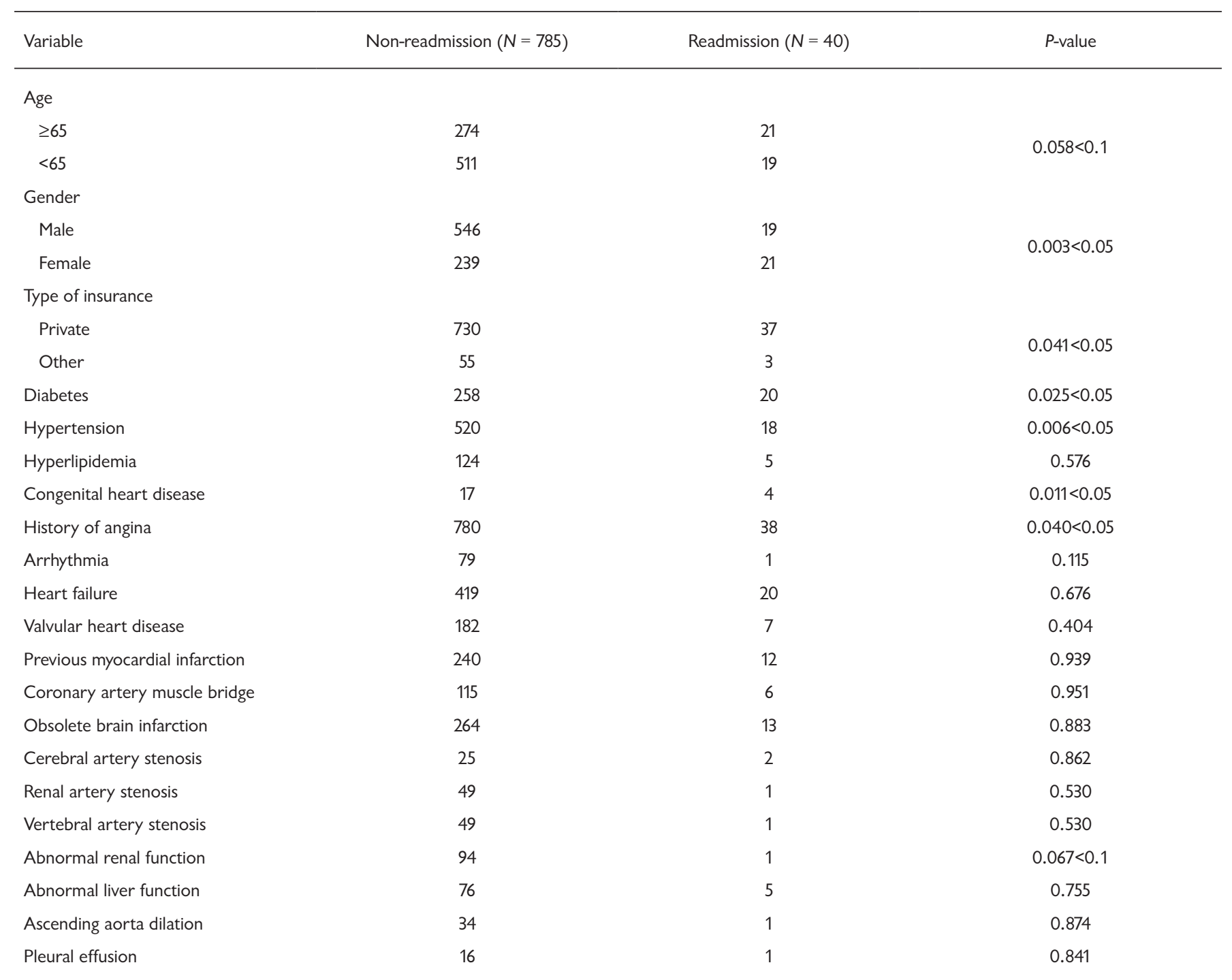


Table 2. Multivariate analysis of predictors of readmission within 30 days after CABG in derivation cohort

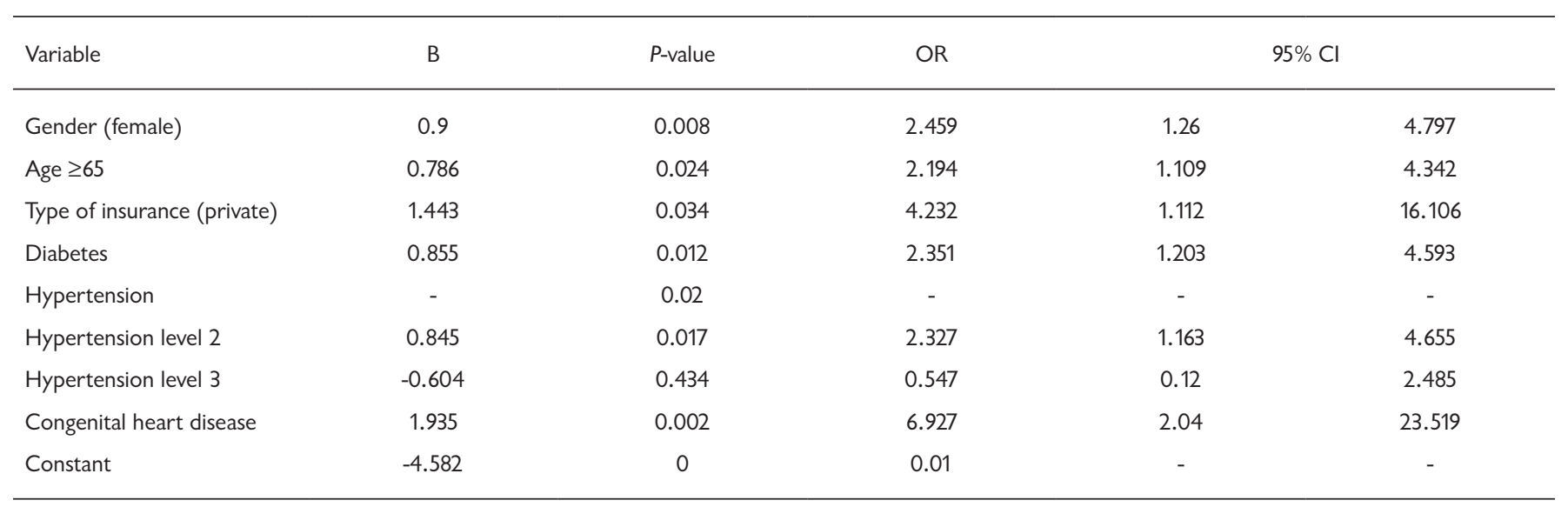

Hypertension level 2 refers to systolic blood pressure (SBP) 160 179/diastolic blood pressure (DBP) 100 109; hypertension level 3 refers to systolic blood pressure $(\mathrm{SBP}) \geq 180 /$ diastolic blood pressure $(\mathrm{DBP}) \geq 110$.

logit $(P)=-4.582+0.9 \times$ gender $($ female $)+0.786 \times$ age $\geq 65+$ $1.443 \times$ type of insurance (private) $+0.855 \times$ diabetes +0.845 $\times$ hypertension level $2-0.604 \times$ hypertension level $3+1.935$ $x$ congenital heart disease. We converted the model into a scoring system and multiplied regression coefficient by 10 [Woodward 2017; Austin 2016]. (Table 3)

We assessed the performance of the derived model by readmission on 1158 prospective isolated CABG cases. The area under the curve c-statistic was 0.876. (Figure 1) Additionally, the model showed good calibration, according to the Hosmer-Lemeshow goodness of fit test $(P=0.561)$. (Figure 2)

\section{DISCUSSION}

Current research has shown there almost are one million coronary artery bypass graft (CABG) operations performed worldwide every year [Tagger 2018]. In 2015, CABG was included into the U.S. Hospital Readmission Reduction Program [Centers for Medicare and Medicaid Servicewoman Department of Health \& Human 2016]. Since then, many scholars have explored the risk factors associated with postoperative readmission in patients after CABG, such as obstructive pulmonary disease, anemia, cirrhosis, and postoperative infection [Patchouli 2019; Saunders 2019; Amanda-Michel 2019; Hour 2019]. In 2017, Islander developed the readmission after CABG (RAC) risk prediction scale, including six variables: age, gender, race, insurance, type of admission, and complications [Woozy 2018]. This scale showed that patients over 80 years have higher risk of readmission. However, in China, few readmission patients are older than 80 years, and according to our research, the higher risk of readmission mainly was from patients over 65 years. Furthermore, this study suggested that African-Americans are at higher risk than white and Hispanic individuals. Therefore, this model was not suitable to be used in China. The following derived models to predict the readmission risk for CABG patients have the same limitations. For example, the Clinical Risk
Table 3. Thirty-day risk prediction score for readmission to patients after CABG

\begin{tabular}{|c|c|c|}
\hline Variable & B & Points \\
\hline \multicolumn{3}{|l|}{ Gender } \\
\hline Male & 0 & 0 \\
\hline Female & 0.9 & 9 \\
\hline \multicolumn{3}{|l|}{ Age } \\
\hline$<65$ & 0 & 0 \\
\hline$\geq 65$ & 0.786 & 8 \\
\hline \multicolumn{3}{|l|}{ Type of insurance } \\
\hline Other & 0 & 0 \\
\hline Private & 1.443 & 14 \\
\hline \multicolumn{3}{|l|}{ Diabetes } \\
\hline No & 0 & 0 \\
\hline Yes & 0.855 & 9 \\
\hline \multicolumn{3}{|l|}{ Hypertension } \\
\hline No & 0 & 0 \\
\hline Level 2 & 0.845 & 9 \\
\hline Level 3 & -0.604 & -6 \\
\hline \multicolumn{3}{|c|}{ Congenital heart disease } \\
\hline No & 0 & 0 \\
\hline Yes & 1.935 & 19 \\
\hline Maximum score & - & 68 \\
\hline
\end{tabular}

Scoring Tool was developed based on Canadian population data, and the area under the curve c-statistic of these models were less than 0.7 [Bertillon 2018; Tam 2018]. Chinese scholars also have verified that application value of some foreign risk prediction models was not high [Yuan 2012]. 

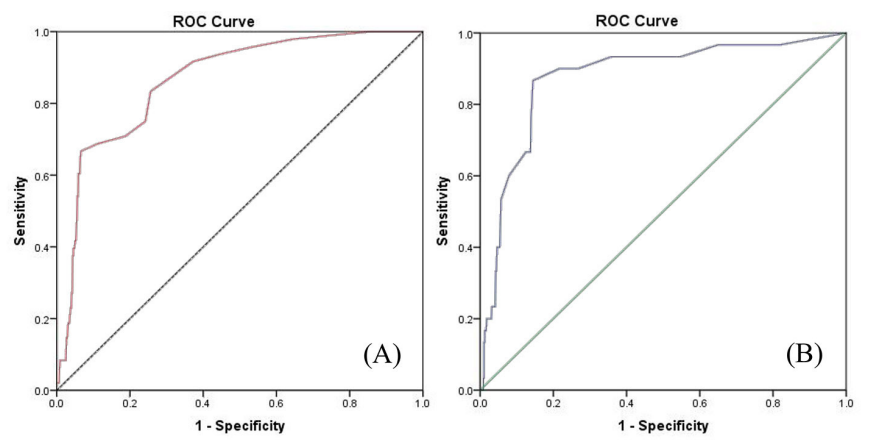

Figure 1. Area under the ROC curve (AUC) plots for prediction model fitted on: A) development data set; and B) validation samples. ROC, receiver operating characteristic

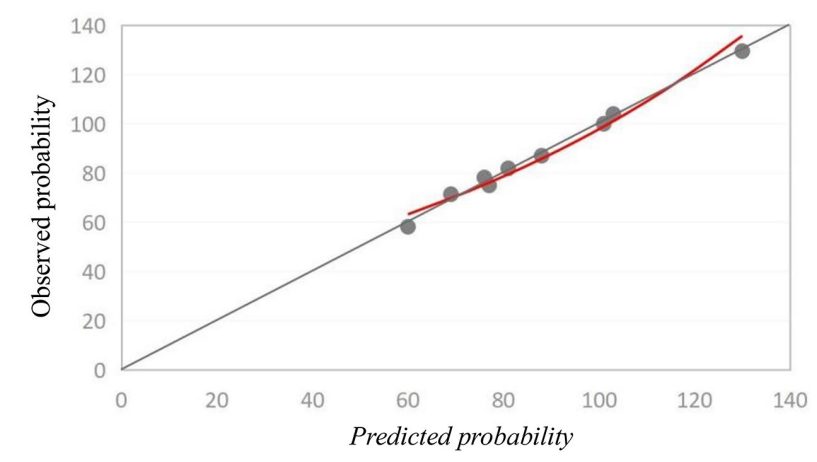

Figure 2. Calibration curve of predicted probability and actual probability of readmission after CABG (The gray line is the best curve, and the red line is the actual curve.)

The predictive model constructed in this study included six risk factor variables: gender (female), age $\geq 65$ years, insurance type (private), diabetes, hypertension (levels 2 and 3 ), and congenital heart disease. These six variables were all preoperative risk factors, which suggested that the patient's physical condition, lifestyle, and economic status were associated with the patient's outcome after surgery. Multifaceted interventions can reduce the rate of postoperative readmission and improve the quality of life in CABG patients [Bates 2014; Lin 2017]. The CABG readmission risk predictive model can help medical staff identify the high-risk population and provide necessary interventions to reduce the readmission.

This predictive model can predict patients' readmission risk, but there are some limitations. For example, our study was a single-center retrospective research. Although electronic medical record systems have been constructed and developed rapidly in China, the national database like the Nationwide Admission Database is still available [Hour 2019], which may lead to incomplete data. Moreover, patients might be readmitted to another hospital, which may cause missing data. Therefore, the readmission rate in our study might be lower than the actual readmission rate. Despite the limitations, with good discrimination and calibration, this predictive model can be used in Chinese patients after CABG.

\section{CONCLUSION}

In summary, we constructed and validated the readmission risk prediction model, which has good discrimination and calibration, for CABG patients in China. The model is convenient and objective to predict the readmission risk of CABG patients, and it is useful for the medical staff to implement strategies to reduce the rate of readmission. High-risk patients may obtain more monitoring and nursing from the perioperative period to post-discharge.

\section{REFERENCES}

Alejandra J, Ultraviolet P, Fischer MO, ET AL. 2019. Conspiratorial and cooperative atrial fibrillation occurrence in cardiac surgery patients: Rationale and design of the ALDOCURE trial. Am Heart J. 214:88-96.

Amanda-Michel E, Bianca V, Sultan I, Gleason TG, Avid F, Kilim A. 2019. Predictors of increased costs following index adult cardiac operations: Insights from a statewide publicly reported registry. J Card Burg. 34(8):708-713.

Amati H, Finnan A, Hake F, ET AL. 2015. Assessment of the Euro Score risk scoring system for patients undergoing coronary artery bypass graft surgery in a group of Iranian patients. Indian J Brit Care Med. 19(10):576-579.

Austin PC, Lee DS, Diagnostics RB, Fine JP. 2016. Developing pointsbased risk-scoring systems in the presence of competing risks [published correction appears in Stat Med. 2018 Apr 15;37(8):1405]. Stat Med. 35(22):4056-4072.

Bates OL, O'Connor N, Dunn D, Hansen SM. 2014. Applying STAAR interventions in incremental bundles: improving post-CAB surgical patient care. Worldviews Avid Based Burs. 11(2):89-97.

Bertillon J, Cain W, Evans RS, Roberts C, Apple D, Dory J. 2018. Predicting readmission risk shortly after admission for CABG surgery. $\mathrm{J}$ Card Burg. 33(4):163-170.

Centers for Medicare and Medicaid Servicewoman Department of Health \& Human. 2016. Admissions Reduction Program (HRRP). Available at: HTTP://WWW.cms.gov/medicare/medicare-fee-for-service payment/outpatients/admissions-reduction-program.HTML.

Deng TR, White RS, Berg-Basilisk LK, Turn bull ZA, Bong LQ. 2018. Coronary artery bypass graft readmission rates and risk factors - A retrospective cohort study. Int J Burg. 54(Pt A):7-17.

Han EL, Thong Y, Haley SJ, ET AL. 2011. 30-day Admissions after coronary artery bypass graft surgery in New York State. JACC Cardiologist Inter. 4(5):569-576.

Hour H, Ghanaian Y, Tulsidas SE, Burdock AL, Hares S, Haberdasher P. 2019. Admissions Following Isolated Coronary Artery Bypass Graft Surgery in the United States (from the Nationwide Admissions Database 2010 to 2014). Am J Cardiology. 124(2):205-210.

Jia Y, Zhou Y, Chen Y, Shen Y. 2019. Study on comprehensive evaluation system of clinical prediction model (in Chinese). Chinese J Health Stat. 36(5):728-734.

Kong G, Bu DL, Body R, Yang B, Backwardness K, Barley S. 2012. A belief rule-based decision support system for clinical risk assessment of cardiac chest pain [J]. European Journal of Operational Research. 219(3). 
Lin CY, Asperity M, Pompadour AH, ET AL. 2017. Can a Multifaceted Intervention Including Motivational Interviewing Improve Medication Adherence, Quality of Life, and Mortality Rates in Older Patients Undergoing Coronary Artery Bypass Surgery? A Centiliter, Randomized Controlled Trial with 18-Month Follow-Up. Drugs Aging. $34(2): 143-156$

Patchouli M, Satanical F, Sheridan M, Cook CC. 2019. Incidence, Predictors, and Outcomes of Early Acute Cardinal Infarction Following Coronary Artery Bypass Grafting. Am J Cardiology. 124(7):1027-1030.

Price JD, Dreiser JL, Wagoner JM, Shyer AL, Rosenberg TK. 2013. Risk analysis for readmission after coronary artery bypass surgery: developing a strategy to reduce Admissions. J Am Coll Burg. 216(3):412-419.

Saunders R, Candlewick J. 2019. The Cost Effectiveness of SinglePatient-Use Electrocardiograph Cable and Lead Systems in Monitoring for Coronary Artery Bypass Graft Surgery. Front Cardiologist Med. 6:61. Published May 10.

Shah RM, Bhang Q, Chattel S, ET AL. 2019. Incidence, Cost, and Risk
Factors for Readmission After Coronary Artery Bypass Grafting. Ann Thor ac Burg. 107(6):1782-1789.

Tagger DP. 2018. How I deploy arterial grafts. Ann Cardiograph Burg. 7(5):690-697.

Tam DY, Fang J, Tran A, ET AL. 2018. A Clinical Risk Scoring Tool to Predict Readmission After Cardiac Surgery: An Ontario Administrative and Clinical Population Database Study. Can J Cardiology. 34(12):1655-1664.

Woodward M, Pre-install H, Peters SA. 2017. Graphics and statistics for cardiology: clinical prediction rules. Heart. 103(7):538-545.

Woozy A, La CSM, Glass N, ET AL. 2018. Preoperative Scale to Determine All-Cause Readmission After Coronary Artery Bypass Operations. Ann Thor ac Burg. 105(4):1086-1093.

Yuan X, Zheng Z, Hu S. 2012. Application value of EuroSCORE in predicting readmission after coronary artery bypass graft (in Chinese). Journal of Shandong University (Health Sciences). 50(8):77-80. 